\title{
Un espacio virtual para vivir la ciencia y la tecnología por medio de streaming
}

A virtual space to experience science and technology through streaming

\author{
Jordan-Andrés Lancheros-González ${ }^{1}$ \\ Magnolia Herrera-Marchena ${ }^{2}$ \\ Pilar-Juliana Morantes-Hernández ${ }^{3}$ \\ Fundación Clínica Shaio - CEMMI \\ Ricardo-Antonio Buitrago-Bernal ${ }^{4}$ \\ Iván-Darío López-Buitrago ${ }^{5}$ \\ Jorge Reynolds Pombo ${ }^{6}$ \\ Fundación Clínica Shaio
}

Recibido: 03.01.2021

Aceptado: 18.01.2021

\section{Resumen}

Considerando las graves consecuencias que ha traído la actual pandemia de COVID-19 a toda escala: económica, social, ambiental, que han llevado a la realización de actividades académicas virtuales, y en aras de mitigar este impacto causado en el ámbito educativo en el

\footnotetext{
1 jordan.lancheros@shaio.com https://orcid.org/0000-0002-5618-7084

2 magnolia.herrera@shaio.com https://orcid.org/0000-0002-5664-1362

3 pilar.morantes@shaio.com https://orcid.org/0000-0002-7395-3019

${ }^{4}$ ricardo.buitrago@shaio.com https://orcid.org/0000-0002-2602-5455

5 ivan.lopez@shaio.com https://orcid.org/0000-0002-9308-5255

6 jorgereynolds@ @otmail.com https://orcid.org/0000-0002-5490-6970
} 
país, se pretende unir esfuerzos para facilitar la continuidad de la educación a través de formación y estimulación enfocadas en el aprendizaje virtual de calidad.

Es por esto que se hace necesaria la implementación de herramientas virtuales por medio de raspberry pi como dispositivo servidor de video (streaming) y manejo de equipos ópticos, como cámaras, para dar respuestas creativas y originales, a las problemáticas del entorno con impacto educativo y social para el país, basado en la utilización de herramientas tecnológicas de desarrollo como método de enseñanza a distancia en una red de área amplia con una presentación continua de video en vivo, para el enriquecimiento de experiencias educativas en Ciencia, Tecnología e Innovación (CTeI). Se requiere con urgencia crear e implementar métodos de enseñanza innovadora en sesiones vivenciales virtuales para la apropiación del conocimiento y el aprovechamiento de la tecnología, además de fomentar la creatividad y el pensamiento propositivo enfocados en el fortalecimiento de un ecosistema rico en generación de ideas como motor dinamizador de una industria vanguardista en pro de mejorar la calidad de vida de los estudiantes de básica y media, atendiendo sus necesidades y realidades.

Teniendo en cuenta lo anterior, se ha implementado un modelo, que contempla y abarca una serie de actividades soportadas en recursos y propuestas pedagógicas de aprendizaje virtuales experienciales por medio de streaming para la observación en vivo de los seres de la naturaleza y su observación en vivo, esto permitirá a los docentes del área de ciencias hacer más eficiente la introducción de nuevas tecnologías en sus procesos de enseñanza, generando en los estudiantes compromiso, despertando curiosidad por la investigación y el emprendimiento, como herramientas para la construcción de un proyecto de vida alrededor del sector de la Ciencia, la Tecnología y la Innovación y de esta manera responder a las necesidades del mundo moderno y consolidar las capacidades del sector educativo en el uso de estas tecnologías.

Palabras clave: Educación, Streaming, Innovación, Sesiones virtuales, Ciencia, Nuevas tecnologías 


\begin{abstract}
The current COVID-19 pandemic brought consequences at all levels: economic, social, environmental, this means there is a need to carry-out educational activities and virtual meetings, mitigating the impact caused in the education policies. For this reason, it is necessary to implement activities in order to facilitate the education continuity through training and stimulation centered in virtual quality learning.
\end{abstract}

For this reason, it is necessary to implement virtual tools through raspberry pi as a video server device (streaming) and management of optical equipment such as cameras for positive and creative responses to resolving educational problems based on the use of technological development tools as a distance learning method in a wide area network with a continuous presentation of live video, for the enrichment of educational experiences in Science, Technology and Innovation

Is necessary to create and implement innovative teaching methods in virtual experiential sessions for the appropriation of knowledge and the use of technology, in addition to promoting creativity and purposeful thinking focus in idea generation.

Based on this, a model has been implement, and contemplates activities supported by resources and pedagogical proposals for experiential virtual learning through streaming for the immersive interaction in various disciplines of science, engineering and technology.

This allowed science teachers to make the introduction of new technologies more efficient in their teaching processes, generating commitment in students, awakening curiosity for research and entrepreneurship, as tools for the construction of a life project around of the Science, Technology and Innovation sector and in this way respond to the needs of the modern world and consolidate the capacities of the education sector in the use of these technologies. 
Keywords: Education, Streaming, Innovation, Virtual sessions science, New emerging technologies

\section{Introducción}

La actual pandemia ocasionada por el COVID-19, trajo consigo una disrupción inminente en los sistemas educativos a nivel mundial; según datos estimados por la UNESCO, más de 1 billón de niños dejaron de asistir a los centros educativos, y pese a que los gobiernos han buscado las alternativas para garantizar la continuidad en la educación, debido a las brechas de inequidad en niveles de desarrollo, se ha hecho evidente que las zonas rurales presentan disparidad en cuanto a la provisión de modalidades de aprendizaje (UNESCO, 2020).

En el caso de Colombia, la presidencia dictó la directiva presidencial n ${ }^{\circ} 2$ del 12 de marzo de 2020, por la cual se indican como principales medidas para evitar el contagio del virus "Trabajo en casa por medio del uso de las TIC; uso de herramientas tecnológicas para comunicarse; hacer uso de herramientas como e-learning, portales de conocimiento, redes sociales y plataformas colaborativas para adelantar los procesos de capacitación y formación que serán inaplazables”

Por su parte la ministra de educación nacional, en la directiva ${ }^{\circ} 06$ de 25 de marzo de 2020 , estableció que para dar continuidad a los programas de Educación para el Trabajo y el Desarrollo humano, se invita a las instituciones que, de manera excepcional, durante el periodo que dure la emergencia sanitaria, ajusten su cronograma de actividades y desarrollen el componente teórico asistido por las herramientas que ofrecen las Tecnologías de la Información y las Comunicaciones TIC, sin que ello implique un cambio en el registro de programa aprobado ni en su modalidad presencial.

Dado lo anterior, y según datos recopilados por la Organización para la Cooperación y el Desarrollo Económicos (OCDE), en Colombia tan solo el 62\% de los estudiantes cuenta con equipos tecnológicos para desarrollar sus actividades escolares (Schleicher, 2020); esta cifra 
posiblemente sea menor debido a que el acceso se limite en el caso de que el recurso deba ser compartido con otros miembros del hogar, sin mencionar que es fundamental contar con espacios tranquilos para el aprendizaje y que el cuerpo docente cumpla con un alto nivel de desarrollo profesional, es por esto que se propende hacia una educación de calidad fortaleciendo los programas educativos, aumentando la cobertura y el acceso a las plataformas digitales, capacitaciones al cuerpo docente y disminución de las tasas de deserción escolar.

\section{Antecedentes}

En la mayoría de los países todavía no existe un uso generalizado de nuevas tecnologías, pero existen docentes que han innovado en esta área, y que están comenzando a incursionar en ella. Uno de los proyectos educativos que se está implementado es el llamado Plan Ceibal. En la publicación El modelo Ceibal. Nuevas propuestas para el aprendizaje, se analizan diferentes aspectos de la educación uruguaya. El mismo tiene 16 capítulos que abordan diferentes cuestiones tales como: los nuevos escenarios educativos, cuál debería ser el rol docente en el siglo XXI, los desafíos en la era digital, la inclusión y la cultura digital y la realidad aumentada en la educación entre otras (Administración Nacional de Educación Pública, 2012).

A nivel de la enseñanza pública, el Consejo de Educación Técnico Profesional (CETP) en ese mismo país ha creado proyectos nuevos como las Unidades Regionales de Educación Permanente, que están trabajando desde el año 2011 con la formación permanente de los docentes (Administración Nacional de Educación Pública, 2012). Desde 2013 estas unidades han desarrollado talleres de acompañamiento a los nuevos docentes que ingresan a la institución y una de las líneas de trabajo son los entornos virtuales y las TIC. En estos encuentros se aplican y demuestran diferentes recursos tecnológicos, entre ellos la Realidad Aumentada aplicada a la educación como forma de incentivar el uso de las nuevas tecnologías en el aula. 


\section{Justificación}

Los desafíos que trajo consigo la actual pandemia condujeron a una transición en los procesos pedagógicos. Si bien las tecnologías de la información y comunicación (TIC) han incrementado su presencia durante la última década en los centros educativos (Martínez \& Tudela, 2020), aún su integración con las prácticas educativas no han alcanzado totalmente los niveles deseados; además si se tiene en cuenta la ruralidad, en donde aún los métodos tradicionales de estudio funcionan, es decir las conferencias cara a cara en un aula, se siguen ofertando, se ve la necesidad de ayudar a cambiar el enfoque pedagógico tradicional.

Es por esto que, el hacer que las instituciones pasen al modo online se hace fundamental, y la mejora del aprendizaje en línea es imprescindible para esta transición, esto también debe incluir la capacitación docente para permitir una adecuada sinergia y un acompañamiento a los estudiantes, puesto que este modelo de aprendizaje trae consigo flexibilidad y autonomía, pero es muy fácil que se caiga en deserción estudiantil por factores que incluyen el entorno familiar, limitaciones en los recursos, cobertura y acceso a red entre otros.

Para tal fin se pretende adoptar el uso de streaming para lograr un aprendizaje inmersivo y de calidad, accesible a todos los niveles que busque mejorar los ecosistemas de entornos digitales y la calidad de aprendizaje.

\section{Desarrollo de competencias}

En Colombia se propone utilizar las líneas de trabajo como son los entornos virtuales y las TIC para hacer frente a los nuevos retos que ha generado el escenario de la pandemia causada por la enfermedad del COVID 19 en la educación. Se proponen las siguientes competencias:

- Independencia cognitiva mediante la metacognición al desarrollar estrategias que concientizan a los estudiantes de su propio aprendizaje en sesiones vivenciales virtuales.

. Desarrollo de habilidades y destrezas para aprender a aprender (aprendizaje autónomo).

Volumen 1. Número 2. Julio - Diciembre 2021 
. Fortalecimiento del pensamiento crítico y reflexivo mediante la construcción de modelos o representaciones del mundo natural propios de su contexto, unido a la producción de representaciones concretas (imágenes, modelos a escala, maquetas, simulaciones)

. Apropiación del trabajo en comunidades científicas.

. Aprendizaje de contenidos conceptuales, procedimentales y actitudinales.

. Concientización de fortalezas y debilidades de tal manera que pueda consolidar un plan de trabajo en donde las primeras sean reforzadas para superar las segundas.

- Aproximación actitudinal y conceptual a la naturaleza de la ciencia al abordar el cómo se han construido los conceptos, teorías y principios de la ciencia.

Para ello se propone el uso de tecnologías de virtualización como streaming para la observación en vivo de los seres de la naturaleza por medio de microscopios ópticos y su visualización en dispositivos móviles inteligentes y software desarrollada para educación en el área de ciencias.

Estas herramientas digitales permitirán no sólo contrarrestar los efectos en la educación que ha provocado la actual pandemia del COVID 19 sino también mejorar los procesos de enseñanza aumentando así el rendimiento académico de los estudiantes y su interés en estos nuevos entornos digitales. Los límites entre el plano físico y el virtual son cada vez más finos,

y la interacción entre ambos proporcionan nuevas herramientas para la formación que hay que saber aprovechar. El plano virtual se ha incorporado inevitablemente al físico y en este sentido se hacen necesarias las tecnologías inmersivas que inciden directamente sobre nuestra visión del mundo.

\section{Desarrollo de la herramienta tecnológica (Streaming)}

Para la implementación de la tarjeta Raspberry pi como medio de servidor de video (streaming) y manejo de equipos ópticos se utiliza esta tarjeta de desarrollo como método de enseñanza a distancia en una red de área amplia provista por una institución educativa en 
Colombia, con una presentación continua de video en vivo (Projects Raspberrypi, s.f.) (figura $1)$.

Pasos para utilizar la tarjeta de Raspberry Pi 3 B+, con un microscopio óptico Leica DMLS y una cámara Pi v.1.3 de $5 \mathrm{Mp}$.

. Reconocimiento de la tarjeta.

. Instalación del sistema operativo (OS).

. Instalación de cámara.

. Configuración de la captura de imágenes o video.

. Configuración de un servicio que permita exportar el video sobre internet.

. Visualización a nivel global.

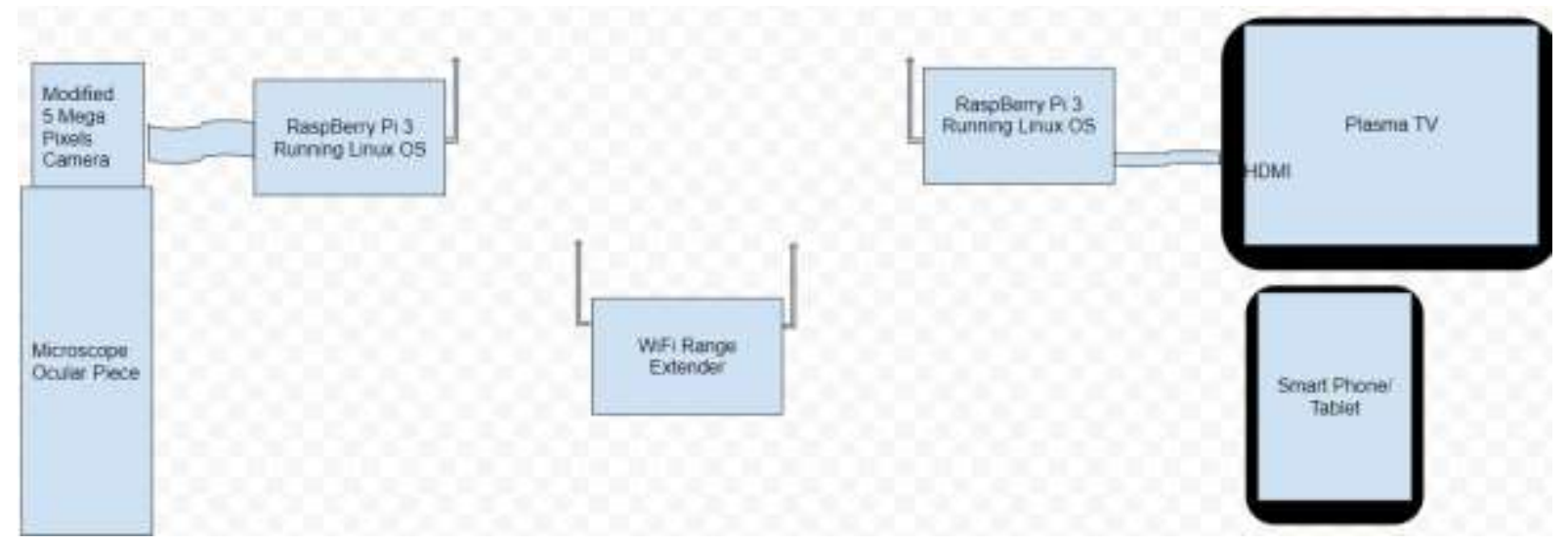

Figura 1 Conectividad de los elementos que componen el sistema de streaming

Fuente: elaboración propia

\subsection{Reconocimiento de la Raspberry}

Se pudo determinar la versatilidad de la tarjeta: Es como tener un computador del tamaño de una tarjeta de crédito. Esta cuenta con una entrada de energía de $5 \mathrm{v}$ a 2,5A que proveerá energía a un procesador Quad Core de 1.2Ghz Broadcom BCM2837 64bit, 4 puertos USB2, 
una memoria RAM de $1 \mathrm{~Gb}$, conectividad a internet por medio de una tarjeta inalámbrica o un conector RJ45 (100 base ethernet). Cuenta con salidas de video compuesto y HDMI (Tabla 1). También se puede conectar una pantalla Touch Screen con conector DSI. La interfaz serie de pantalla (DSI) es una especificación de la Alianza de la interfaz de procesador de la industria móvil (MIPI) destinada a reducir el costo de los subsistemas de pantalla en un dispositivo móvil. Está comúnmente dirigido a LCD y tecnologías de visualización similares (qastack, s.f. ). Define un bus serie y un protocolo de comunicación entre el host (fuente de los datos de imagen) y el dispositivo (destino de los datos de imagen). Para el audio cuenta con la salida estero de 4 polos (figura 2).

La placa de la cámara Raspberry Pi 3 se conecta directamente al conector CSI de la Raspberry Pi. Es capaz de ofrecer una imagen nítida con una resolución de 5MP o una grabación de video HD de 1080p a 30 fps. Diseñada y fabricada a medida por la Fundación Raspberry Pi en el Reino Unido, la placa de cámara Raspberry Pi cuenta con un sensor Omnivision 5647 de 5MP (2592x1944 píxeles) en un módulo de enfoque fijo. El módulo se conecta a Raspberry Pi 3, a través de un cable plano de 15 clavijas, también conocido como cable flexible, y se conecta al zócalo ZIF 15 de montaje en superficie, a la interfaz en serie de cámara (CSI) MIPI (interfaz en serie de cámara de interfaz de procesador industrial móvil) dedicada, que fue diseñada especialmente para interconectarse con cámaras a nivel industrial. El bus CSI es capaz de velocidades de datos extremadamente altas y transporta exclusivamente datos de píxeles al procesador BCM2835. La placa en sí es pequeña, de alrededor de $25 \mathrm{~mm}$ x $20 \mathrm{~mm}$ x $9 \mathrm{~mm}$, y pesa poco más de $3 \mathrm{~g}$, lo que la hace perfecta para aplicaciones móviles u otras aplicaciones donde el tamaño y el peso son importantes. El sensor en sí tiene una resolución nativa de 5 megapíxeles y tiene una lente de enfoque fijo a bordo. En términos de imágenes fijas, la cámara es capaz de imágenes estáticas de 2592 x 1944 píxeles y también admite grabación de video de 1080p a 30 fps, 720p a 60 fps y 640x480p 60/90. La cámara es compatible con la última versión de Raspbian, el sistema operativo preferido de Raspberry Pi 


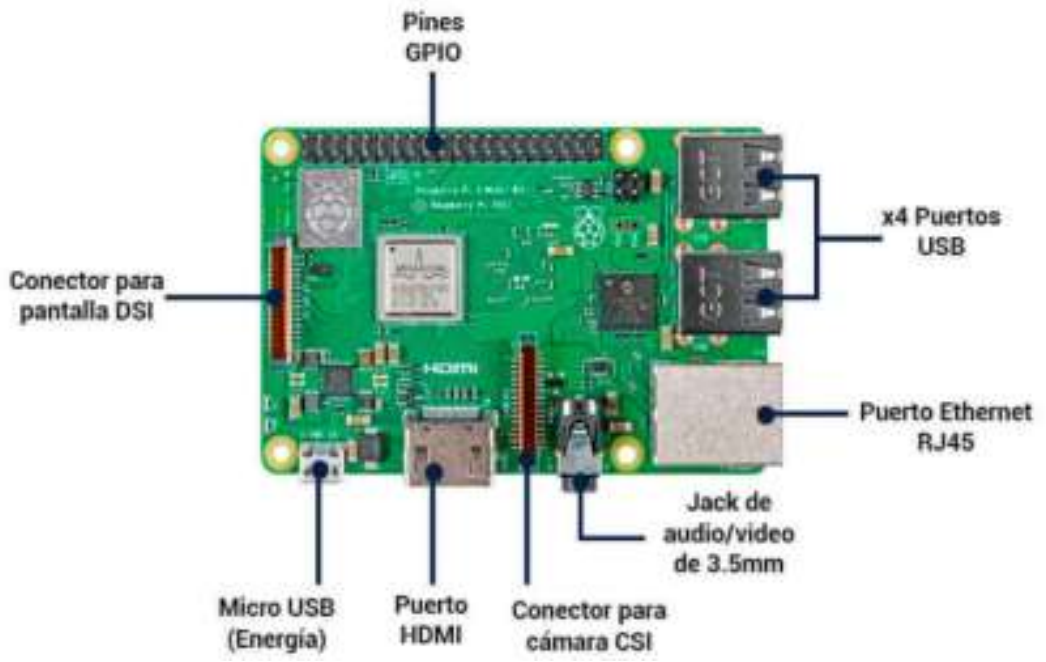

Figura 2. Visualización física de la tarjeta y sus conectores

Fuente: Mecatrónica Uno, 2018

\begin{tabular}{ll}
\hline CANT & DESCRIPCIÓN \\
\hline 1 & Quad Core 1.2GHz Broadcom BCM2837 64bit CPU \\
1 & 1GB RAM \\
1 & BCM43438 wireless LAN and Bluetooth Low Energy (BLE) on \\
& board \\
1 & 100 Base Ethernet \\
40 & pin extended GPIO \\
4 & USB 2 ports \\
4 & Pole stereo output and composite video port \\
1 & Full size HDMI \\
1 & CSI camera port for connecting a Raspberry Pi camera \\
1 & DSI display port for connecting a Raspberry Pi touchscreen \\
1 & display \\
1 & Micro SD port for loading your operating system and storing data \\
\hline
\end{tabular}

Tabla 1. Especificaciones de Raspberry pi

Fuente: (Raspberrypi, s.f.) 


\subsection{Instalación de sistema operativo}

Para la administración de la Raspberry Pi3 se instaló un sistema operativo por medio de una microSD. En la actualidad hay alrededor de 10 pero el más usado es el Raspbian, un sistema operativo libre basado en la distribución de Linux denominada Debian y optimizado para el hardware de Raspberry Pi.

La instalación incluye una preparación del repositorio eliminando cualquier tipo de dato y formato de la memoria. Luego, podemos instalar Raspberry Pi Imager en Windows, Linux y MacOS. Una vez dentro, descargamos el archivo binario de instalación para nuestro sistema operativo. Seleccionamos el sistema Raspbian que deseamos: Si el usuario prefiere la interfaz gráfica (conocida también como GUI del inglés graphical user interface), tiene la posibilidad de usar el mouse o ratón, como puntero para señalar y seleccionar los diferentes elementos de la GUI, que tradicionalmente se categorizaron como ventanas, iconos o carpetas. Consume un poco más de espacio, pero para el aprendiz es la forma más amigable de acercarlo al dispositivo.

La instalación también se puede realizar en modo CLI (interfaces de línea de comando o Command Line Interface por sus siglas en inglés). Esta interface es para usuarios más avanzados y para los cuales sus aplicaciones no requieren ni siquiera un monitor para la visualización del proceso, pues todo lo pueden hacer vía remota.

Para nuestro caso se hará a través de GUI.

\subsection{Instalación de cámara}

La cámara tiene la cinta de comunicación que debe ser conectada al zócalo ZIF15. Se debe tener en cuenta que tiene un "derecho" para su conexión. En la figura 3 (A y B) se puede ver que el final de la cinta tiene una parte azul. La otra "punta" esta soldada de fábrica a la tarjeta que contiene la cámara. Esta parte es una protección que permite la fácil introducción de la 
cinta en el zócalo evitando que esta se doble al presionarla hacia la placa. Dicha parte azul, debe quedar de frente al puerto RJ45 (conexión de red por cable).

Se debe verificar que quede completamente alineada con la placa y después de ello, se presionan los seguros del zócalo para que la cinta quede firmemente conectada impidiendo que se salga de su sitio al momento de hacer uso de ella (Higinio, 2018b).
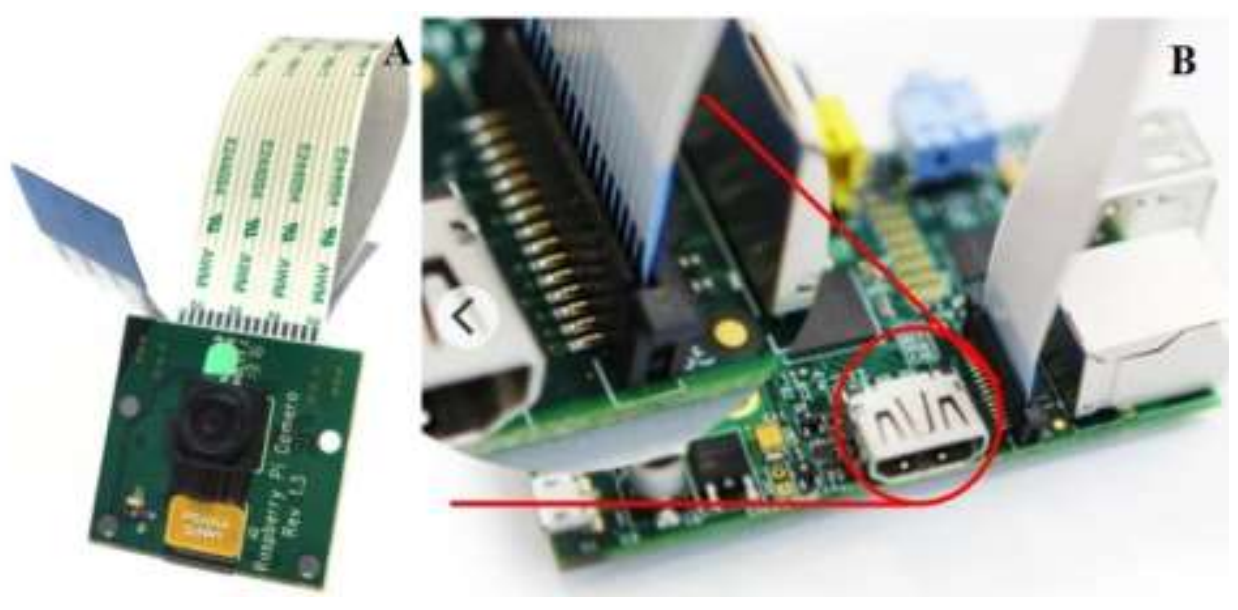

Figura 3. Instalación de la cámara. A. Cámara Pi v. 1.3. B. Posición de la cinta en el zócalo Fuente: Pi Supply.

The maker Emporium, 2021

\subsection{Configuración de la captura de imágenes o video.}

Se realizó una modificación al método de observación de la cámara a través del microscopio y posteriormente se adaptó al portaocular, para definir la visualización correcta de la cámara al ser retirado el lente panorámico que trae enfrente del sensor. Esto nos permitió tener una observación plana de la imagen que será potenciada por los lentes objetivos del equipo óptico. Al encontrar la distancia focal apropiada, se fija al tubo binocular.

Es importante habilitar la cámara desde la configuración de Raspberry PI. La interfaz por defecto esta desactivada. En este punto se cambió de su estado a “Activada” y el sistema debió ser reiniciado para que tome los cambios. Desde este punto en adelante, la captura de imágenes o video se realizó por medio de la Interfaz de Línea de Comandos (CLI), esto se hizo 
presionando las teclas $\mathrm{Ctrl}+\mathrm{Alt}+\mathrm{T}$. Inmediatamente generó la pantalla de comandos llamada en raspbian LX Terminal.

Para probar el funcionamiento de la cámara se ingresaron los siguientes comandos:

. raspivid -o testvideo07.h264 -t 6000. Este comando en específico permite crear un video llamado Testvideo07 el cual estará en un formato tipo h264 y tendrá una duración de 6 segundos.

. raspistill -o Desktop/image.jpg. En este caso la cámara toma una foto y la salva en el Escritorio con el nombre de Image en formato .jpg La resolución por defecto será de 2592x1944 (biblioman09, 2012; The Post apocalyptic inventor, 2016; Carles , 2020) (figura 4 A-B).

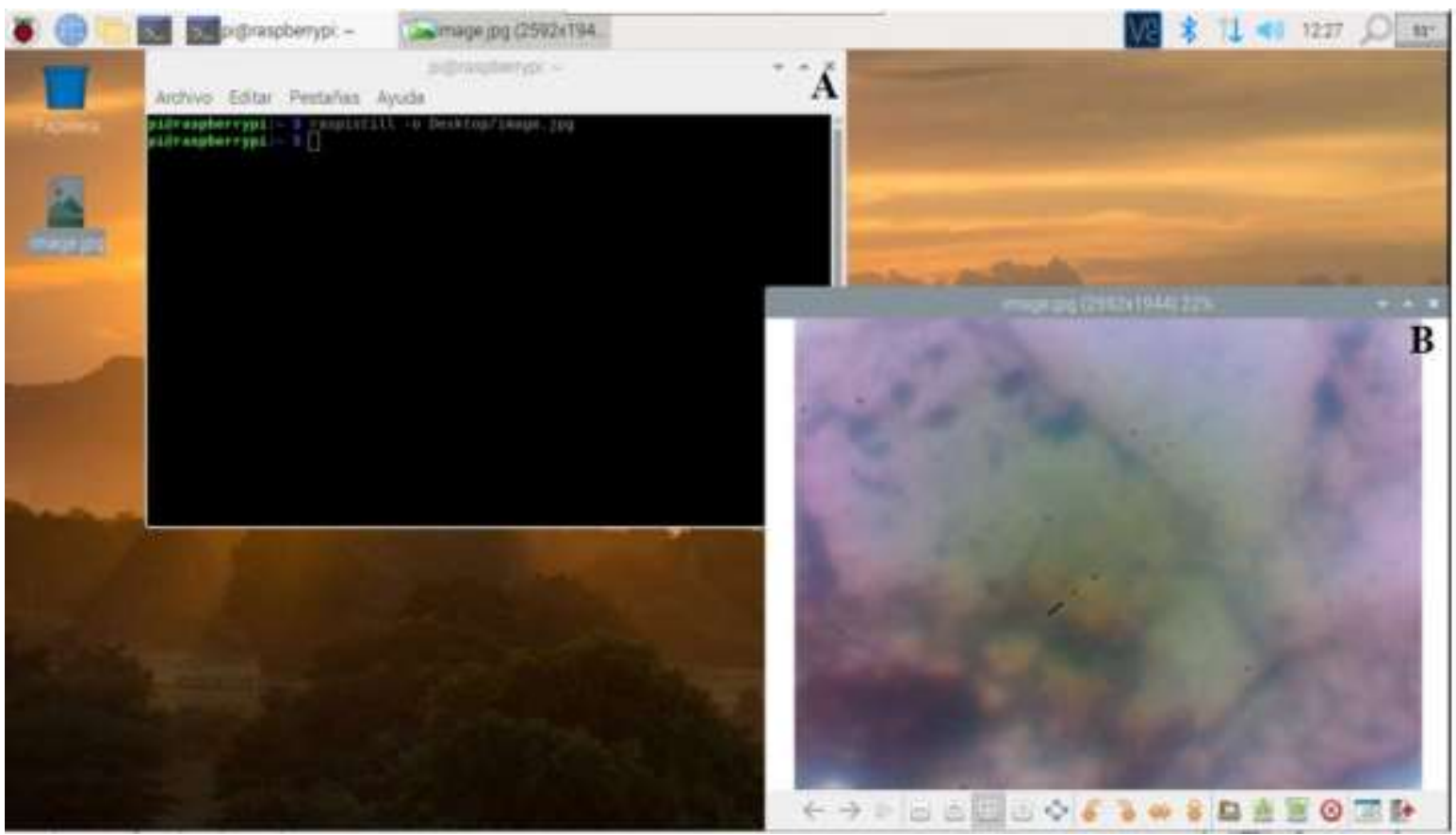

Figura 4. Escritorio de Raspberry pi con terminal de comando.

A. Comandos de archivo de imagen. B. Microfotografía de lámina de roca sedimentaria

Fuente: elaboración propia 


\subsection{Configuración de un servicio que permita exportar el video sobre internet.}

Para este paso en particular se tomaron diferentes alternativas:

. Instalación de un servicio LAMP y Web Server para la transmisión de video mediante streaming.

. Instalación de Python-flask

. Inscripción al Servicio NoIP para salida directa a Internet.

\subsection{Reconfiguración del Servicio de internet Local}

Se realizó la instalación y configuración del servidor Apache, con la creación de carpetas donde se alojará el servicio HTML que será presentado a la comunidad educativa.

Apache es una aplicación de servidor web popular que puede instalar en Raspberry Pi para permitirle servir páginas web. Por sí solo, Apache puede servir archivos HTML a través de HTTP. Con módulos adicionales, puede servir páginas web dinámicas utilizando lenguajes de programación como PHP.

. Instalación de PHP, este es un procesador, un código que se ejecuta cuando el servidor recibe una solicitud de una página web a través de un navegador web. Calcula lo que se debe mostrar en la página y luego envía esa página al navegador. A diferencia del HTML estático, PHP puede mostrar contenido diferente en diferentes circunstancias. PHP es un lenguaje muy popular en la web: grandes proyectos como Facebook y Wikipedia están escritos en PHP.

. Instalación de MariaDB. Es un motor de base de datos popular. Al igual que PHP, se usa ampliamente en servidores web, razón por la cual proyectos como WordPress lo usan y por qué esos proyectos son tan populares.

- Instalación de los paquetes MariaDB Server y PHP-MySQL ingresando el siguiente comando en la ventana del terminal: "sudo apt-get install mariadb-server php-mysql-y" 
Todas estas instalaciones fueron dadas para las posibles entradas a la página web y registros de las mismas en una base de datos.

Se instaló Python3 con una aplicación llamada Flask. Con este software y unas entradas adicionales en la cámara y haciendo una sencilla página en HTML, se logró reproducir video en vivo para la red local. Este streaming apuntaba a una dirección IP fija en la Raspberry, que al ser digitado en el buscador de cualquier dispositivo (celular, tableta o computador), muestra la imagen de lo que estaba ocurriendo en la platina del microscopio. Se observó la muestra casi sin retraso (delay) en el recorrido a través de los ejes X, Y y Z.

Para que la entrega de esta imagen fuera de la Red de Área Local (Local Area Network LAN por sus siglas en Ingles), se optó por un servicio gratuito llamado NoIP. Este pretende, tomar la dirección IP dinámica del proveedor de servicios de Internet abriendo unos puertos específicos en la tabla de enrutamiento asignados a la dirección IP privada (de lado del usuario) otorgada por el ISP (Internet Service Provider) y traducirla a un sistema de nombres de dominio (Domain Name System o DNS, por sus siglas en inglés). El DNS es un sistema de nomenclatura jerárquico descentralizado para dispositivos conectados a redes IP como Internet o una red privada. Este sistema asocia información variada con nombres de dominio asignados a cada uno de los participantes. Su función más importante es traducir nombres inteligibles para las personas en identificadores binarios asociados con los equipos conectados a la red, esto con el propósito de poder localizar y direccionar estos equipos mundialmente (Loredo, 2017).

De igual forma, se utilizó un router para poder hacer la traducción de direcciones de red, también llamado enmascaramiento de IP o NAT Network Address Translation), debido a que la dirección IP usada era del segmento privado(casa). NAT es un mecanismo utilizado por routers IP para cambiar paquetes entre dos redes que asignan mutuamente direcciones incompatibles. Consiste en convertir, en tiempo real, las direcciones utilizadas en los paquetes transportados. El tipo más simple de NAT proporciona una 
traducción una-a-una de las direcciones IP. La RFC 2663 se refiere a este tipo de NAT como NAT Básico, también se le conoce como NAT una-a-una. En este tipo de NAT únicamente las direcciones IP, las sumas de comprobación (Checksums) de la cabecera IP y las sumas de comprobación de nivel superior, que se incluyen en la dirección IP, necesitan ser cambiadas. El resto del paquete se puede quedar sin tocar (al menos para la funcionalidad básica del TCP/UDP, algunos protocolos de nivel superior pueden necesitar otra forma de traducción). Es corriente ocultar un espacio completo de direcciones IP, normalmente son direcciones IP privadas, detrás de una única dirección IP (o pequeño grupo de direcciones IP) en otro espacio de direcciones (normalmente público) (Pascual, 2017).

En ninguna de las opciones anteriores funcionó la prueba externa por los protocolos que tiene el ISP actualmente.

\subsection{Visualización a nivel global}

Ante el impedimento de las opciones anteriores surgió una tercera aplicación: RemoteIt.

Remote.it virtual private internet (VPI) es una solución de red y comunicación segura que brinda los beneficios de una VPN sin la necesidad de dispositivos VPN o una administración de seguridad complicada. Simplemente instalando el pequeño software en la Raspberry Pi, un registro en mi propio VPI y cierra todos los puertos que puedan ser atacados por piratas informáticos, incluidos 22 (SSH), 80 (HTTP) y 443 (HTTPS).

Instalando en el sistema operativo Raspbian con el comando: "sudo apt install remoteit "se pudo avanzar en la configuración de la aplicación. Cada uno de estos comandos tardo menos de un minuto en completarse mientras descargaron los archivos necesarios.

Posteriormente se abrió un navegador y se escribió find.remote.it en la barra de URL y se presionó la tecla Enter. Al utilizar la GUI de Pi directamente, puede utilizar el navegador de Pi. 
Si la conexión al Pi es usando SSH a través de la LAN, se puede copiar la URL a un navegador en su PC local. Ahora se puede acceder al panel de administración web de remoteit siguiendo las instrucciones. Esto permitió que el software instalado en la Raspberry hiciera enlace con la página web. De ser necesario, ellos proporcionarán un localizador del dispositivo solo presionando un botón llamado find.remote.it.

La página provee unos códigos de acceso al servicio que previamente se ha escogido, el cual para nuestro caso fue Http. EL software generó los enlaces seguros sin necesidad de intervención del ISP, modificación en el Router, Apertura de puertos que pudieran vulnerar la seguridad de la red, etc (Higinio, 2019a).

La prueba se hizo con personas distribuidas a través de la ciudad de Bogotá y un usuario en Alaska con una visualización del movimiento de la muestra petrográfica ubicada en la platina del microscopio.

\section{Análisis y discusión de resultados}

A partir de los resultados preliminares, se pudo establecer que, a partir de la utilización de una tarjeta de desarrollo, que se puede convertir fácilmente en un computador, amplió la posibilidad de adquirir tecnología a bajo costo para ser entregada a la comunidad educativa, a través de la creación de habilidades técnicas, de pensamiento, lógicas y de implementación en nuevas tecnologías y software.

La Raspberry permite una ampliación del conocimiento en otros sistemas operativos diferentes a Windows cómo normalmente lo identificamos, ya que se pueden tener desde versiones de Linux (una de ellas ajustada a la tarjeta con el nombre de Raspbian), o para el manejo de puertos GPIO para el control de Internet de las Cosas (IoT por sus siglas en inglés) como Windows 10 IoT Core o para arquitecturas como ARM como lo es SARPi. En total 10 Sistemas operativos para una misma tarjeta.

Volumen 1. Número 2. Julio - Diciembre 2021 
Sin embargo, a pesar de la versatilidad de los muchos sistemas podría darse la dificultad en las aulas de encontrar los docentes que operen dichos sistemas. El aprendizaje no es complicado, solo toma un poco más de tiempo, como todo lo nuevo al iniciarse, para lo que se requerirán capacitaciones.

La pandemia ha dejado al descubierto grandes falencias en el campo educativo, al no poder llegar a todos y cada uno de los estudiantes, sobre todo en áreas rurales, donde los niños habitualmente hacen largas travesías para llegar a su colegio, tomar las clases y emprender de nuevo trayectos que podían durar horas. Si el país, las gobernaciones y sus alcaldías hacen la implementación de las Raspberry en sus programas, es posible que se les proporcione un impulso para incrementar el conocimiento tecnológico en el país distribuido en los estudiantes que reciban estas tarjetas de desarrollo. De igual forma podrán llevarse a casa con la idea de que dichos sectores del gobierno implementen redes con o sin internet (mesh), puesto que, ellas, sus estudiantes y docentes, serán generadores de conocimiento comunitario

Cabe la posibilidad de que el gobierno no esté interesado en proveer cambios que serían disruptivos en la sociedad. Este, como dueño de las estructuras, requiere de inversión para la instalación de redes de área amplia en las zonas urbanas y apartadas desde donde surgen los estudiantes que con anhelo van a sus clases cuando no hay pandemia. Estas redes tienen unas especificaciones que van desde los $10 \mathrm{Mb}$ hasta los $30 \mathrm{Mb}$ (cuando se desean hacer transmisiones en vivo como se ha visto en los ensayos). Estos estándares están clasificados según la FCC. Por ejemplo, si te encanta hacer streaming de contenido 4K, necesitarás 25 Mbps como mínimo (Federal communications comission, 2018; Verizon , 2021).

\section{Conclusiones}

La observación en vivo por medio de herramientas como streaming a través de microscopios ópticos, fortalecerá a niños y adolescentes de educación básica y secundaria un interés innato además de cultivable en generaciones de nuevos científicos, biólogos e ingenieros que puedan plantear preguntas y soluciones a los problemas planteados en investigación. Ya que tendrán

Volumen 1. Número 2. Julio - Diciembre 2021 
la oportunidad de interactuar con una herramienta que permite dicho crecimiento intelectual sin importar el estrato socioeconómico.

Formación de un pensamiento crítico, lógico y dirigido a solución de problemas en el estudiante.

Incentivar la investigación en etapas tempranas de estudio.

Romper las barreras de la distancia y de tecnología ya que el colegio estaría entregando estas tarjetas de desarrollo a los estudiantes con la posibilidad de conexión a una red de área amplia con o sin conexión a internet.

\section{Referencias}

Administración Nacional de Educación Pública. (2012). El modelo ceibal. Nuevas tendencias para el aprendizaje. Uruguay : Centro CEIBAL - ANEP.

biblioman09. (2012 de octubre de 2012). Webcam server con Raspberry Pi [video]. Obtenido de https://www.youtube.com/watch?v=1yGi7_bWbFU\&amp\%3Bt=992s

Carles , J. (14 de marzo de 2020). Instalar Raspbian con Raspberry Pi Imager de forma sencilla. Obtenido de https://geekland.eu/instalar-raspbian-con-raspberry-pi-imager/

Directiva No. 06. (25 de marzo de 2020). Uso de las tecnologías en el desarrollo de porgramas de educación para el trabajo y el desarrollo humano. Ministra de educación nacional.

Directiva presidencial No. 02. (12 de marzo de 2020). Medidas para atender la contingencia generada por el COVID-19, a partir del uso de las tecnologías de la información y las telecomunicaciones $\quad$-TIC-. Obtenido de 
https://dapre.presidencia.gov.co/normativa/normativa/DIRECTIVA\%20PRESIDENCIAL\% 20N\%C2\%B0\%2002\%20DEL\%2012\%20DE\%20MARZO\%20DE\%202020.pdf

Federal communications comission. (6 de febrero de 2018). Guía de Velocidades de Banda Ancha. Obtenido de https://www.fcc.gov/consumers/guides/guia-de-velocidades-de-bandaancha

Higinio, H. (15 de octubre de 2018b). Sistema de Seguridad Raspberry Pi - Sensor de Movimiento y Camara HD con envio de imagenes a correo[video]. Obtenido de https://www.youtube.com/watch?v=rK6uLwMRtIs\&t=245s

Higinio, H. (6 de enero de 2019a). Web Server Video Streaming con Raspberry Pi - Muy facil [video]. Obtenido de https://www.youtube.com/watch?v=fCTc1sBQwi8

Ligarretto, R. (2020 de mayo de 2020). Educación virtual: realidad o ficción en tiempos de pandemia. Obtenido de Pesquisa Javeriana: https://www.javeriana.edu.co/pesquisa/educacion-virtual-realidad-o-ficcion-en-tiempos-depandemia/

Loredo, R. (5 de Julio de 2017). Conexiones remotas con Raspberry PI, usando SSH, VNC, SFTP [video]. Obtenido de https://www.youtube.com/watch?v=hVGO2XSPXbQ

Martínez, J. M., \& Tudela, A. (2020). Interactions between virtual spaces and schools: a collective case study. Future Internet, 1-10.

MecatrónicaUno. (18 de septiembre de 2018). Raspberry pi, tutoriales. "Hola Mundo" con Raspberry Pi 3. Recuperado el 5 de enero de 2021, de https://mecatronicauno.com/holamundo-con-raspberry-pi-3/ 
Pascual, J. (29 de diciembre de 2017). Domótica Barata (8). Instalación Raspbian y servicio No-IP (\#4K) [video]. Obtenido de youtube.com/watch?v=XkiN16vwOt4

Pi Supply. The maker Emporium. (2021). Raspberry Pi Camera Board v1.3. Obtenido de https://uk.pi-supply.com/products/raspberry-pi-camera-board-v1-3-5mp-1080p

Projects Raspberrypi. (s.f.). Build a LAMP Web Server with WordPress. Obtenido de Download WordPress: https://projects.raspberrypi.org/en/projects/lamp-web-server-withwordpress $/ 5$

qastack. (s.f. ). Conector de video DSI. Obtenido de https://qastack.mx/raspberrypi/512/dsivideo-connector

$\begin{array}{lllllll}\text { Raspberrypi. } & \text { (s.f.). Raspberry } & \text { Pi } & \text { Model } & \text { B. Obtenido de }\end{array}$ https://www.raspberrypi.org/products/raspberry-pi-3-model-b/

Schleicher, A. (2020). The impact of COVID-19 on education insights from education at a glance 2020. OECD.

The Post apocalyptic inventor. (20 de noviembre de 2016). Hack: Digital Microscope with $\begin{array}{lllll}\text { Raspberry } & \mathrm{Pi}+\text { PiCam } & + & \text { Obideo]. }\end{array}$ https://www.youtube.com/watch?v=M6TvCOPN5pI

UNESCO. (2020). Coalición Mundial para la Educación COVID-19. Obtenido de Global education coalition.

Verizon. (2021). Ancho de banda. Obtenido de https://espanol.verizon.com/info/definitions/bandwidth/ 
REVISTA INTERNACIONAL DE PEDAGOGÍA E INNOVACIÓN EDUCATIVA |

Volumen 1. Número 2. Julio - Diciembre 2021 ISSN: 2745-0341 (En línea) 